\title{
First VLBA Images of the Nuclei in 3CR Lobe-dominated Quasars
}

\author{
D. H. Hough \\ Trinity University, Department of Physics, San Antonio, TX 78209, U.S.A.
}

R. C. Vermeulen

Netherlands Foundation for Research in Astronomy, Dwingeloo, The Netherlands

A. C. S. Readhead

Caltech, Mail Code 105-24, Pasadena, CA 91125, U.S.A.

\begin{abstract}
We report the first VLBA images of the nuclei in eight objects from the $3 \mathrm{CR}$ complete sample of 25 lobe-dominated quasars (LDQs): 3C 207, 3C 208, 3C 212, 3C 245, 3C 249.1, $3 \mathrm{C} 263,3 \mathrm{C} 270.1$, and $3 \mathrm{C} 275.1$. These images reveal core-jet structures of considerable extent and complexity. In combination with earlier VLBI work on the 3CR LDQ sample, the partial distribution of apparent parsec-scale jet speeds for ten objects is relatively flat up to $\sim 5 h^{-1} c$, or perhaps even $\sim 10 h^{-1} c$. While this is not inconsistent with simple beaming models and quasar-FR II radio galaxy unification, it is crucial to obtain the complete speed distribution for truly definitive tests. Work is in progress on multi-frequency, dual-polarization, and phasereferencing VLBA observations on selected objects in the sample.
\end{abstract}

As part of a long-term study to test relativistic beaming models and AGN unification scenarios (e.g., Hough 1994), we have begun using the VLBA to image the nuclei in the 25 lobe-dominated quasars (LDQs) from the 3CR complete sample (Laing, Riley, \& Longair 1983). Since these objects qualify for this flux-densitylimited sample on the basis of their lobe emission alone, yet have nuclei strong enough to image with VLBI, they comprise a practical sample with minimal orientation bias that is well-suited for these tests.

Thus far, we have imaged eight relatively strong ( $>50 \mathrm{mJy}$ ) LDQ nuclei with the VLBA. We observed four objects at $8.4 \mathrm{GHz}$ in 1995 September3C 207, 3C 208, 3C 212, and 3C 249.1-and another two at $15 \mathrm{GHz}$ in 1996 February-3C 270.1 and 3C 275.1. In 1996 June, we obtained multi-frequency $(5,8.4$, and $15 \mathrm{GHz})$, dual-polarization data on $3 \mathrm{C} 245$; to date, we have made an $8.4 \mathrm{GHz}$ total intensity image of this object. As part of an ongoing special collaboration by Hough, Zensus, \& Porcas (1996), 3C 263 was imaged at 5, 8.4, and $15 \mathrm{GHz}$ from observations made in 1995 November.

These new images reveal one-sided, core-jet structures of greater extent and complexity than we had generally found in earlier VLBI studies of LDQ nuclei. While a resolved core or close double $(<1$ mas in size) dominates, faint jet features out to $\sim 2-5$ mas and beyond are found in every source; in $3 \mathrm{C} 245$, we confirm the essentially continuous, faint jet out to $\sim 25$ mas reported by Hough et al. (1996). There are indications of mild jet curvature in a number of sources, and in the case of $3 \mathrm{C} 207$ (see Figure 1) there is strong curvature with jet components ranging over $\sim 20^{\circ}$ in position angle relative to the core. The VLBI jets all have the same "sidedness" as the large-scale VLA jets (e.g., Bridle et al. 1994), and the mean axes of the VLBI and VLA jets are well-aligned. We note that the multi-frequency imaging of $3 \mathrm{C} 263$ clearly shows that the presumed compact core has a flatter spectrum than the multiple extended jet components.

In combination with our previous VLBI studies of the 3CR LDQ sample, we can at present construct a partial distribution of apparent parsec-scale jet speeds 
(or limits) for ten objects. There are ambiguities in component identifications at different epochs that render some speeds quite uncertain. However, it does seem clear that the observed distribution is fairly flat up to $\sim 5 h^{-1} c$, or perhaps even $\sim 10 h^{-1} c\left(H_{0}=100 h \mathrm{~km} \mathrm{~s}^{-1} \mathrm{Mpc}^{-1}, q_{0}=0.5\right)$. This result is not inconsistent with simple beaming models and unification of core- and lobe-dominated quasars with FR II radio galaxies. However, in the absence of a few highly superluminal speeds that would rule out simple beaming models, stringent tests (including possible implications for $H_{0}$ ) await speed measurements for the remaining fifteen LDQ nuclei-most of which are relatively weak $(<50 \mathrm{mJy})$ - to see if they have superluminal speeds in the $\sim 1-3 h^{-1} c$ range as predicted. To this end, we recently (1997 January) made phase-referencing VLBA observations of nine LDQ nuclei in the $\sim 5-30 \mathrm{mJy}$ range and have detected fringes on two of the three sources checked to date.

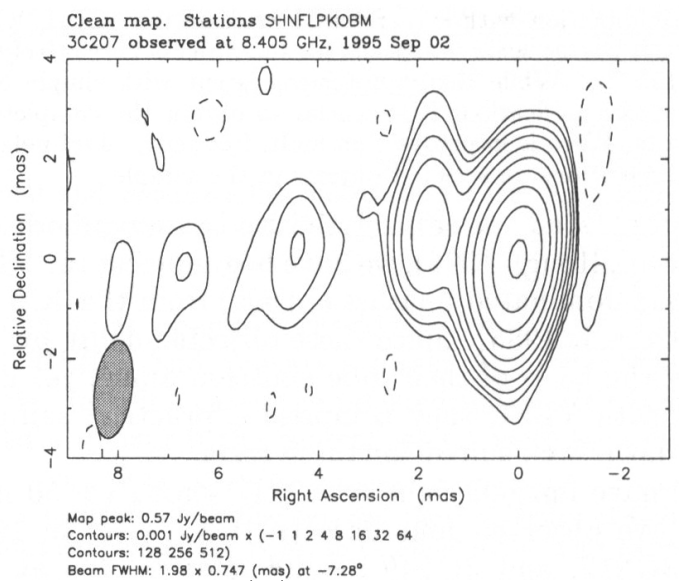

Figure 1. VLBA image of the nucleus of the lobe-dominated quasar 3C 207.

Acknowledgments. DHH is grateful for support under NSF grant AST-9422075, and wishes to acknowledge the significant contributions to this work by Trinity undergraduates Erika Barth, Philip Beyer, Lara Cross, and Ellen Phifer. The National Radio Astronomy Observatory is a facility of the National Science Foundation, operated under a cooperative agreement by Associated Universities, Inc.

\section{References}

Bridle, A. H., et al. 1994. $A J, 108,766-820$.

Hough, D. H., 1994. in Compact Extragalactic Radio Sources, eds. J. A. Zensus \& K. I. Kellermann (Green Bank: NRAO), 169-174.

Hough, D. H., et al. 1996. ApJ, 459, 64-72.

Hough, D. H., Zensus, J. A., \& Porcas, R. W. 1996. ApJ, 464, 715-723.

Laing, R. A., Riley, J. M., \& Longair, M. S. 1983. MNRAS, 204, 151-187. 